\title{
Conflict Resolution: Analysis of the Existing Theories and Resolution Strategies in Relation to Face Recognition
}

\author{
Akeem Alabi $^{1}$, Babajide Afolabi ${ }^{2}$, Bernard Akhigbe ${ }^{2}$, Adewole Ayoade ${ }^{1}$ \\ ${ }^{1}$ Department of Computer Science and Engineering, Oduduwa University, Ipetumodu, Nigeria \\ ${ }^{2}$ Department of Computer Science and Engineering, Obafemi Awolowo University, Ile-Ife, Nigeria
}

\section{Email address:}

newalabikompurer@gmail.com (A. Alabi)

\section{To cite this article:}

Akeem Alabi, Babajide Afolabi, Bernard Akhigbe, Adewole Ayoade. Conflict Resolution: Analysis of the Existing Theories and Resolution Strategies in Relation to Face Recognition. American Journal of Computer Science and Technology. Special Issue: Facial Disparity. Vol. 2, No. 4, 2019, pp. 52-59. doi: 10.11648/j.ajcst.20190204.12

Received: November 13, 2019; Accepted: November 26, 2019; Published: December 24, 2019

\begin{abstract}
A scenario known as conflict in face recognition may arise as a result of some disparity-related issues (such as expression, distortion, occlusion and others) leading to a compromise of someone's identity or contradiction of the intended message. However, addressing this requires the determination and application of appropriate procedures among the various conflict theories both in terms of concepts as well as resolution strategies. Theories such as Marxist, Game theory (Prisoner's dilemma, Penny matching, Chicken problem), Lanchester theory and Information theory were analyzed in relation to facial images conflict and these were made possible by trying to provide answers to selected questions as far as resolving facial conflict is concerned. It has been observed that the scenarios presented in the Marxist theory agree with the form of resolution expected in the analysis of conflict and its related issues as they relate to face recognition. The study observed that the issue of conflict in facial images can better be analyzed using the concept introduced by the Marxist theory in relation to the Information theory. This is as a result of its resolution strategy which tends to seek a form of balance as result as opposed to the win or lose case scenarios applied in other concepts. This was also consolidated by making reference to the main mechanisms and result scenario applicable in Information theory.
\end{abstract}

Keywords: Conflict Resolution, Conflict Theory, Facial Images, Disparity, Expression, Recognition

\section{Introduction}

The face is naturally considered as the most important part of the body crucial for identity as well as exhibition of feelings; a scenario not easily replicated using computers due to factors such as expression, distortion, occlusion and other disparity-related issues. Expression remains a key non-verbal means of communication of emotions vis-à-vis the state of mind which is a frequent occurrence in humans and therefore takes the lead when discussing disparity. In addition, expression mostly leads to deformation (i.e. a twist or wrap in the shape of a facial component) which in turn could bring about occlusion (i.e. a blockage or coverage of a particular facial component) as shown in figure 1. One gray area of necessity in this regard is the philosophy behind the various forms of expressions in terms of cause(s) of their existence and the possible message they are meant to convey. On this, certain components of the face do interact in a form of dependence to actualize the intention of the individual in question. What first comes to mind during facial analysis has to do with the status any possible-numeric form a face image can take especially when dealing with the one with disparityrelated issues. This becomes a great concern due to the existence of a binding force among the various components making up a face. The point here is that, once there is a change in the status of a set of images of the same face, a contradiction is expected to ensue either against the recognition of such a face or the intended message associated the face's status; a phenomenon referred to as "conflict". Although, the term conflict has not been frequently employed in this area of research, some of the motives or intentions behind certain expressions by humans indicate some form of game-playing gestures which could properly be addressed using conflict-related concepts. One motivating fact related to this is that research has proven that people are often motivated to subvert identification by manipulating their 
appearance thus posing issues related to disguise and impersonation which are game-playing scenarios associated with conflict [1]. Several research theories have existed in the pursuance of providing answers to conflicting subjects in relation to human endeavours. In extension, certain sustainable progresses have been recorded in the application of these theories in aspects of wars, truce, divide, difference and the likes. Despite the existing convergence in the resolution strategies of most conflict theories, applying such to the face recognition scenario requires a different approach in terms of analysis because of the divergent views associated. This paper is therefore aimed at examining the various theories available in the analysis of conflict with a view to identifying with relevant justification, which of them largely agrees with providing the needed recognition mechanism and measure in a conflict scenario involving face images.
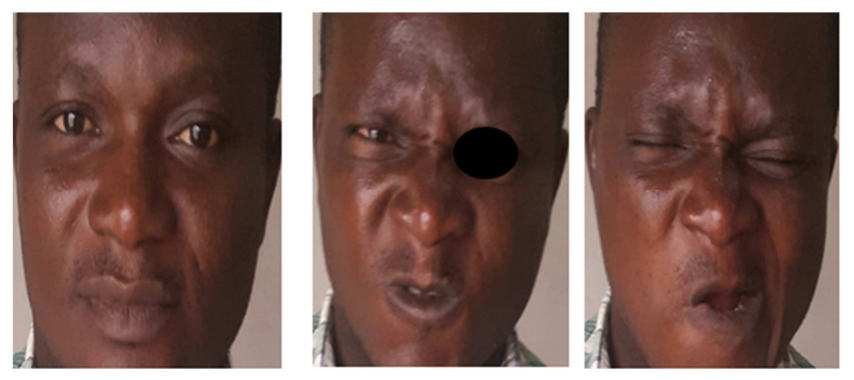

Figure 1. Diagram showing neutrality, occlusion and deformation in faces.

\section{Related Works}

\subsection{General Overview}

The term "Conflict Resolution" may be explained as the process (technique, procedure and tools) of providing actionable, affordable and unbiased solution to opposing or contradicting scenarios. It can also be defined it as the managing of conflict and negotiation of a solution in that respect [2]. Conflict analysis and resolution play some important roles in our day-to-day existence. Areas such as business, administration, labour, management, law suits etc. are usually addressed using some form of conflict resolution strategies [3].

Several forms of discussions have emerged in face recognition on conflict-related issues with different models proposed even though, were not addressed as conflict. For instance, an attempt was made to address the issues related to expression deformation and interpersonal deformation using three-dimensional faces [4]. Patterns of expression were first learnt with the aid of Principal Component Analysis (PCA) and employed to morph out deformation as it relates to expression. The outcome proved effective on Face Recognition Grand Challenge Version 2.0 (FRGC V2.0) database with superior recognition performance. A novel framework was introduced over the deformation and lighting insensitive metric to determine the dense correspondence between image and Neire Bayes' Classifier is applied to improve recognition [5]. The whole thing was to solve the problem of lighting and expression variations and the result obtained proved promising.
An estimation of the level of disparity resulting from camera resolution in selected images was also determine with methodology involving the capturing of some images in full form and the determination of their depth information warped into image position as a form of disparity followed by the application of some required steps [6]. The result showed correctness as regards to the determination of disparity and texture map of used images. There was also the need to improve on the Eigen-selection for better recognizing the features of African bust being art work of the face. An approach known as variable-size Eigenvectors selection for image representation was employed with a view to address the disparity between a typical human face image and that of an African bust [7]. The technique represents an improvement of the existing Principal Components Analysis and thus, proved efficient in the analysis and recognition of African bust images. Problems associated with two-dimensional images were addressed with an idea to analyze the possible challenges of two 2D-images captured from two different cameras and resolve the disparities between them [8]. The images in question were first converted into their three-dimensional format with the aid of 3D binocular disparity technique and the recognition scenario in both cases were said to be positive with a given range of viewing angles. Issues related to the extension in error rates as regards to the problems of variable lighting, pose, facial expression, aging and inaccurate alignment had also been addressed; a study which brought about many benefits such as the use of geometric depth information rather than color and texture (i.e. Invariant to lighting conditions), ability to rotate face model in three-dimensional spaces (Invariant to head angles), 3D modules captured to scale (absolute measurement Invariant to camera distance) [9]. This was as a result of the newly used $3 \mathrm{D}$ cameras which allow subsecond generations of $3 \mathrm{D}$ face model. A model was also developed to test the efficiency of the Eigenface algorithm on images with plain features in comparison with those with distinct features [10]. The result showed that recognition of faces with distinct features took the lead compared to those images with plain features. A study carried out about an aspect of disparity related to hyper-spectra image classification due to the complexity and non-linearity of the data involved showed resulting output surpassed which both Principal Components Analysis Technique (PCA) and Linear Discriminant Analysis (LDA) performance-wise [11]. In a survey conducted on facial expression approaches with Local Binary Pattern (LBP), Local Gradient Code (LGC) and Local Directional Pattern (LDP), whereby the LBP only exhibits a comparison mechanism of the central pixel value within the neighboring value while the relationship among the pixel values within the neighbors was the case in the LGC, results showed that the LGC outweighed the LBP and LDP in which an image in its LDP form is being divided with blocks of histogram to generate the refined features [12]. Other areas of disparity as far as researches in face recognition include efficient disparity estimation-form stereo images using hybrid guided filter, facial expression recognition with Convolutionary Neural Network coping with few data and training sample order, Utilization of a disgusting 
image data set to understand and predict visual disgust and Effect of rotation and scaling of images [13-16]. A combination of Convolutionary Neural Network and specific image pre-processing steps were also employed to demonstrate the variance in features in the way people show expression with better results obtained [14]. Finally, a technique known as Scale-Invariant Feature Transform (SIFT) algorithm was developed and Generalized Hough Transforms (GHT) employed in addition to find the rotation and scaling of an image. This was as a result of the need to address a form of condition related to conflict due to image rotation.

\subsection{Notable Conflict Theories and Their Resolution Strategies}

Conflict resolution, in terms of theories and applications, cut across diverse areas of human endeavours. Popular concepts in this include Karl Marx Theory, Game Theory concept (e.g. Prisoner's Dilemma, Penny Matching Problem, Chicken Problem etc.) and the Lanchester Theory.

\subsubsection{Karl Marx Theoretical Concept}

Generally speaking, a society is said to be composed of people or individuals of different status with different means of livelihood, hence, posing some form of differences in the area of resources sharing. The implication of this is that people in possession of resources will protect and keep away what they either have or is/are available to them while those in need will surely try to have those resources or share from those in control of such resources. This often brings about a constant struggle between the rich and the poor [17]. This theory introduced by Marx isn't about whether or not conflict is bad or not but to understand its scenarios and provide measures towards addressing it with a view to having stable situation or condition of living for people within a given society. The concept here provides answers to questions as well as way-out about avoidable situations such as wars, violence, unrests, revolutions and the likes. The theory has been applied to the handling of conflict issues in finance or related aspects. Strategies involved in this resolution include resources re-allocation between the rich and the poor, mediating in other manner between these two parties and so on; a strategy that usually brings about the introduction of progressive taxes, minimum wages, incentives, social assistance and the likes. The idea is to narrow the gap between affected parties in order to prevent uncontrollable occurrences capable of causing unrest or war.

\subsubsection{Prisoner's Dilemma}

The concept in the analysis of the Prisoner's Dilemma remains one of the most famous problem solving strategies employed in addressing issues related to conflict. Prisoner's Dilemma is an example of a non-zero sum game; in other words, the "win" status of a player doesn't necessarily amount to the "lose" status of the other player [18]. This has been presented in diverse form of research applications. In details, two suspects arrested for a certain crime were put to a game tactic with a view to ensuring that they get prosecuted even though the evidence available against them wasn't enough. Meanwhile, the police had to create a work around hence came up with the following conditional possibilities; defect and cooperate. To cooperate in this case means "keep quiet" and therefore both suspects are expected not to say anything. On the other hand, "defect" has to do with "talking to the police" at least in a manner to incriminate one another [18]. The established rules against each of the suspects (in his separate location) are as follows;

Rule 1 (Applicable to both suspects): Cooperate (i.e. remain silent) and serve short sentences.

Rule 2 (Applicable to either of the suspects): If one of the suspects defects (i.e. betrays the other), he gains his freedom and the other serves a long term in jail.

Rule 3: If both defect (i.e. betray one another), each one gets a lesser sentence.

The elements in the payoff matrix (Table 1) were drawn from these three rules; with elements $(-5,-5)$ denoting "cooperate" since they both get shorter sentence if they cooperate (i.e. remain silent) and $(-10,-10)$ representing the "defect" whereby both suspects are assumed to have chosen to defect fetching them lesser sentences (each) as compared with when only one defects. Finally $(0,-15)$ is about one of the suspects betraying the other. The illustrations above shows that the suspects are likely to go for the "defect" option and so, bringing up their best outcome at $(-10,-10)$ of the same is rationally played only once even though it is clear (result-wise) that their expected best outcome is to cooperate.

Table 1. Suspects' moves and Payoffs matrix.

\begin{tabular}{lll}
\hline & Cooperate & Defect \\
\hline Cooperate & $(5,-5)$ & $(0,-15)$ \\
Defect & $(0,15)$ & $(-10,-10)$ \\
\hline
\end{tabular}

\subsubsection{The Penny Matching Problem}

The term "penny" shares meaning with the token-like material (just as in a dice form) with two directly opposite side outcomes Head and Tail. In the concept of game theory, penny matching (Figure 2) involves two players placing pennies simultaneously on a table with outcomes (payoff) depending on whether or not the pennies match [19]. If the pennies match, the first player wins and takes the other's penny. On the other hand, the second player takes the first player's penny when the pennies don't match. The scenario in the penny matching is considered zero-sum since one player's loss is tantamount to the other player's gain [19]. Also, the game requires that each player is entitled to only one choice of the penny status (i.e. either Head or Tail). Hence, there is not any room for uniformity of results. In other words, one of the players must surely lose or win i.e. No win-win or lose-lose situations. Penny matching game is a way of demonstrating how rational decision-makers seek to maximize their pay-offs. The scenario in Table 2 shows two players (P1 and P2) with their possible payoffs in matrix form. The first of the four sets of numbers shown in the given cells represented belong to P1 while the second belongs to P2. Also, a "win" is denoted by "+1" while "-1" represents 
"loss". The outcome $+1,-1$ in cell1 is about Head-Head (i.e. $\mathrm{P} 1$ wins). This is simply because the two pennies match making player 1 the winner hence, P1 takes P2's penny while P2 loses the penny. The instances in cell 2 (i.e. $-1,+1$ ) bring about the reverse situation. In this case the Head-Tail outcome portrays player 2 as the winner since the pennies do not match; hence, $\mathrm{P} 1$ loses his penny to $\mathrm{P} 2$. The whole table only consists of those two outcomes i.e. P1 wins while P2 loses (as shown in cells 1 and 4) and P2 wins while P1 loses (as shown in cells 2 and 3). However, outcomes such as "win-win" i.e. $(+1,+1)$ or "lose-lose" i.e. $(-1,-1)$ are never

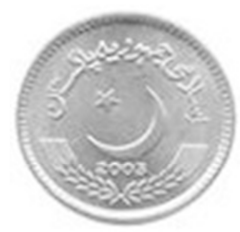

a

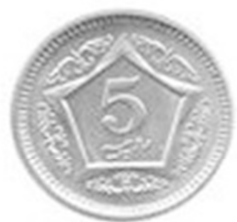

H,T

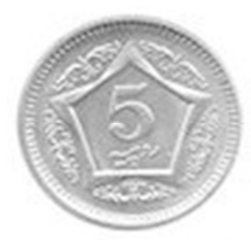

C

\begin{abstract}
$\mathrm{T}, \mathrm{T}$
\end{abstract}

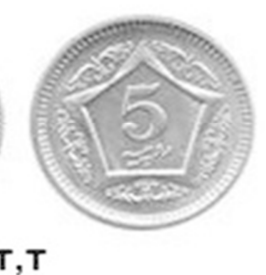

Figure 2. Diagram illustrating Penny Matching. obtainable in the penny matching game theory [19].

Table 2. Payoff matrix for penny matching.

\begin{tabular}{lll}
\hline P1/P2 & Heads & Tails \\
\hline Heads & $+1,-1$ & $-1,+1$ \\
Tails & $-1,+1$ & $+1,-1$ \\
\hline
\end{tabular}

\subsubsection{Chicken Problem}

The Chicken Problem (Sometimes written as Chicken's Problem) consists of players with interactions covered by the Game Theory Concepts.

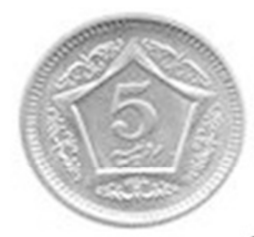

b

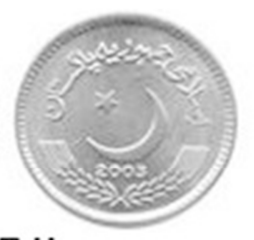

$\mathbf{T}, \mathbf{H}$

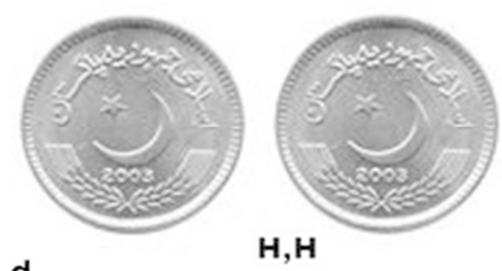

d

The scenario in this concept (Figure 3) presents a special form of game with unique payoffs from the actors involved. Unlike in most games whereby two players exhibit their strategies in a manner that eventually leads to one player winning, the conflict here is about one player directly opposing the other leading to either bigger conflict or cancellation of the existing conflict [18]. Two conflicting players tend to be moving towards each other until they either crash or one of the players surrenders and so moves out of the way. In game terminologies, whenever both players move away, the winner (i.e. the opposing party) gets the pride/glory while the loser (i.e. the opposed) gets the embarrassment. Technically, situation like this simply brings about difficulties in quantifying of the possible payoffs needed to proceed with required analysis. In other words, ascribing or assigning value(s) to results such "gain" and "loss" might not really be feasible enough to make deductions. However, negative payoff emerged whenever none of the opposing parties moves; a situation that usually leads to the term known as "crash". The term crash in this context is tantamount to severe situation of conflict and so, negative outcome(s) are expected as payoffs. A real-life example of this was the confrontation involving the United States of America (USA) and the then USSR on the USA's rejection of USSR military presence in environs close to them by forming blockage against the USSR troops on the way to such places which eventually ended by the discontinuation of the journey by the USSR to avoid escalation.

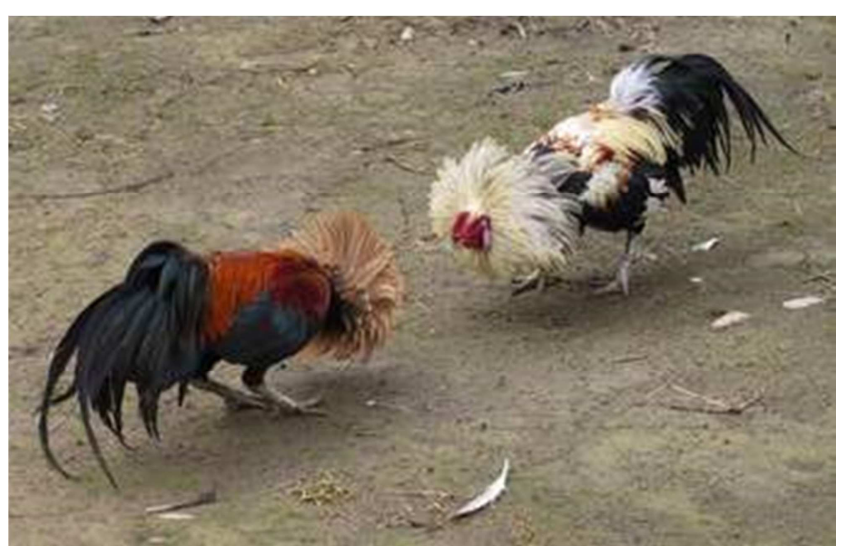

Figure 3. The Chicken Problem.

\subsubsection{Lanchester Theory}

The Lanchester theory applies to a set of two linear ordinary differential equations in a way to establish and comprehend the dynamics of a conflict between two opposing parties. In this case, two opponents fight each other with sizes or number of resources considered as state variables [20]. However, there is dependence between the size of the forces and their per capita effectiveness measured by individual attrition rates and the decrease of the said forces over time. The theory was modeled in different forms namely the Lanchester Linear Law for ancient war and his Square Law for the modern war. Meanwhile, progressive analysis taking into consideration 
issues related to implications of information superiority for ground combat brought about a third law known as mixed law [20]. One way of explaining the difference between the ancient warfare and that of the modern is about the possibility of one party having an advantage over the other due to its larger committed force. For instance, there could be invariably unidentical engagement between a party 1 with minimal force and another say "party 2" with larger force (probably with higher number of fighters) in the ancient warfare. In this, victory or success is really a function of the number of people in a particular fighting party. This isn't the case in the modern warfare in which case, a party with larger fighters might not enjoy some form of advantage over the other thus determining who wins in the end may not be a function of size or number of fighters. However, merging these two scenarios earlier mentioned brought about the Lanchester third law also known as The New Lanchester Law [20]. One of the simplest mathematical models proposed by Lanchester involving two sides of strength $\mathrm{x}(\mathrm{t})$ and $\mathrm{y}(\mathrm{t})$ are described as follows;

$$
\begin{gathered}
\frac{d x}{d t}=-\mathrm{ay}(\mathrm{t}), \mathrm{x}(0)=\mathrm{x}_{0} \\
\frac{d y}{d t}=-\mathrm{bx}(\mathrm{t}), \mathrm{y}(0)=\mathrm{y}_{0} \\
\frac{x_{0}-x(t)}{y_{0}-y(t)}=\frac{a_{x}}{b_{y}}
\end{gathered}
$$

Where 1 and 2 represent individual combat on the other hand with 3 as the equation of state [21].

\section{Discussions}

\subsection{Deduction from the Review}

The issue of conflict (theory-wise) revolves around terms such as contradiction and/or opposition born out of some form of differences thus giving rise to several interpretations especially with respect to the various resolution strategies existing. The analysis of the existing conflict concepts in a way, poses certain questions especially with regards to their applicabilities in relation to face recognition. For instance, two facial images of an individual (say $f_{1} \& f_{2}$ ) are said to be in conflict with each other provided that the correlation existing between them is negative in value. This brings about different approaches towards evaluating possible dissimilarities among features in question and thus, one might be curious to clearly understand if most (if not all) theories of conflict directly or indirectly agree with and address the conflict scenario observed in the analysis of missing facial components as a likely condition responsible that may compromise the individual's identity. Detailed study of the existing theories as explained earlier prompts to the fact that some concepts in some of these theories tend to differ in terms of results/resolutions with respect to the the desired result expected if they were to be applied to the conflict scenario in the analysis of facial images. Therefore, as a key aspect of this research work, there is need to effectively determine the suitability of some of these existing theories in this area of study. This shall be analyzed by navigating through the following questions and their possible responses;

a. What really constitutes conflict in face recognition?

b. What are the possible players involved with the respective stance of each of them during conflict and;

c. What is the resolution expected to change?

\subsection{Conflict Scenario in Face Recognition}

As shown in figure 4, conflict scenario in face recognition has to do with the dissimilarity between two facial images of the same individual as a result of the effect of disappearance of one or more key components (features) of a particular facial image. In essence, conflict is expected to occur from the possible contradiction of opinions emanating from a strange status of an image as a result of dsitortion in its number of key components. This scenario of conflict could be born out of unforseen occurences (such as accident, deformation, and so on.) which might be grouped as intentional or unintentional. For instance, losing a part of the face due to accident or related instances is never a function of the persons state of mind and so, considered 'unintentional'. On the other hand, an individual may choose to take up or stage actions with a view to hiding or compromising his/her own identity. In reality, the act of hiding/covering one's eye or another feature of the face undertaken by ill-minded people while perpetrating evils is in a way to create an identity different from the actual being. This scenario of conflict is an example is definitely 'intentional'.

\subsubsection{Possible Players Involved and Respective Stance During Conflict}

Conflict in this context of face recognition involves two facial images of the same person as the main parties involved. The scenario could also be explained as consisting of two sets of facial features each of different images but of the same individual. This is because of the fact that an image is made up of certain key features thus forming a set of features.

Every feature of the face has a complimentary role (Information) played in the exhibition of the expected expression associated with any possible emotion. Therefore, the absence of a feature is tantaumount to the loss of certain information which is likely responsible for some level of mis-representation of the individual in question. In terms of stance, the distorted facial image in this case which has to be processed in relation to an already trained and stored image of the same person poses a form of compromise in relation to the identity of the person. In this same vein, a facial image with incomplete key features is treated as a potential source of contradiction vis-à-vis the one in a face space (i.e. the set of trained image(s) of the said individual). 


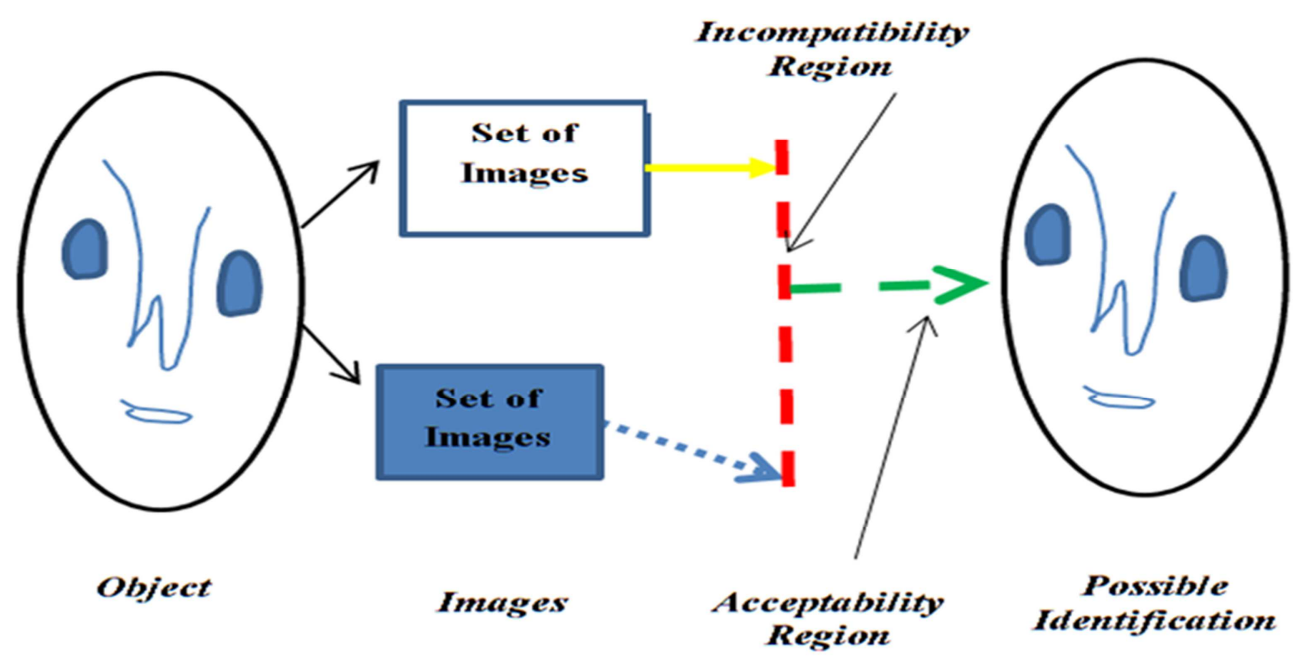

Figure 4. Conflict Scenario in Face Recognition.

\subsubsection{Expected Impact of the Resolution}

The possible contradiction (feature-wise) is a function of the factor responsible for it. As earlier stated, this could be intentional and/or unintentional as the case may be and as an effect, it stands to portray the identity of the person in question in a different manner; a condition referred to as false identity is likely to come up. As a measure towards addressing this, the required resolution is expected to create a form of balance inbetween any inconsistency emanating from this conflict by bringing up a template which is possible of harnessing features of a given test image of a face (the one with distortion) in relation to those of a trained image (unaffected image) with a view to identifying the person irrespective of the distortion.

\subsection{Juxtaposition of the Conflict Theories}

Although, the facial conflict scenarios under 'intentional' present patterns that follow the same as those of a game, the resolution strategies applied in most of the existing game theory concepts seem to be in one direction. This owes to the fact that the idea of the results obtained or expected in each of its known concepts explain the need to have a winner in any form of conflict between the parties involved. Two of these concepts that seem to totally agree with this "win scenario" in terms of the outcome of resolution are "the Chicken Problem" and "the Penny Matching". In each of these, strategies are put in place to ensure a player wins in the end. In a likely manner of approach, the various optionsresult strategies such as "Betray - get reduced sentence (Jailterm)" and "Silent - get equal jail-term" as presented in the "Prisoner's dilemma" somehow give rise to a bi-direction form of resolution. In essence, it is all about either one party/player looses (remain silent and get more sentence than expected) while the other wins (betray and get reduced sentence) or both loose (Both remain silent and get reduced sentence both). However, not all conflicting issues are expected to end up in a form of "win" or "lose" outcome solution-wise. The case in face recognition isn't about whether a distorted image to be put to test gets rejected or accepted as resolution; but requires the mechanism that could present a unifying platform capable of mediating between the face images in question irrespective of their differences since they emanate from the same person.

As stated in Karl theory (Marxist theory), resolving conflicts sometimes requires an approach that could bring about a balance between parties involved. In other words, a resolution doesn't necessarily have to proclaim a player as the "win" party since resolution usually tends to bring about justice (i.e. a balance of status) which isn't always about winning or losing. This is evident in the case of the rich (enough resources) and the poor (limited resources) where part of the resolution is to obtain a form of concensus inbetween the two parties with a view to maintain the stability of the region or society. Logically speaking, the resolution parameters in this case are expected to be a function of some inputs from both the rich as well as the poor. This concept employed in karl theory also finds its trend in the analysis of conflict-related issues using Information theory (IT) and so, stands out in terms of the needed strategies and expected result required for addressing the said conflict scenario related to facial features. The Information theory which is considered 'the pioneer theory in this regard' also gives credence to the resolution concept proposed in the Marxist theory. Strategies in IT are put in place to provide answers to questions such as "By what formalism should prior knowledge and proposed mechanism be combined with incoming data to draw formally justifiable inferences in resolving conflict?". The idea is to devise a measure to process the elements of distorted image with respect to those of trained images with a view to resolving any possible differences thus recognising the said individual. This showcases how the Marxist and IT concide in terms of strategies as well as expected results in the analysis of any conflict scenario in face images.

\subsection{Expected Resolution Strategy}

With some form of conflict-related disparities existing 
between the set of trained images and those of the test images (Figure 5), it is obvious that requirement (A) may not be as exact as those of $(B)$, hence, calling for measures to establish a form of balance between the two sets of facial images. As earlier discussed, the resolution strategy is to create the needed mapping function by harnessing the differences in the available requirements of the images in play thus making possible for achieving uniform status with a view to achieving the desire balance. This balance serves as the resolution entity and so makes the identification of the said individual possible. The idea is to make sure the status of the test image conforms to the identity of the individual in question irrespective of the distortion.

\section{Conclusion}

In this paper, a number of theories and their respective concepts that could be applied in resolving conflict in relation to facial recognition have been proposed with various strategies discussed with a view to determining which of them best aligns with desired outcome in the area of conflict emanating from facial feature disparities. In line with this, aspects such as key features of a face and the relationships existing among them were discussed in relation to how they affect one another in conflict situation especially when there is distortion or disappearance of one or more features of a face. The study observed that the issue of conflict in facial images can better be analyzed using the concept introduced by the Marxist theory in relation to the Information theory. This is as a result of its resolution strategy which tends to seek a form of balance as result as opposed to the win or lose case scenarios applied in other concepts. This was also consolidated by making reference to the main mechanisms and result scenario applicable in Information theory.

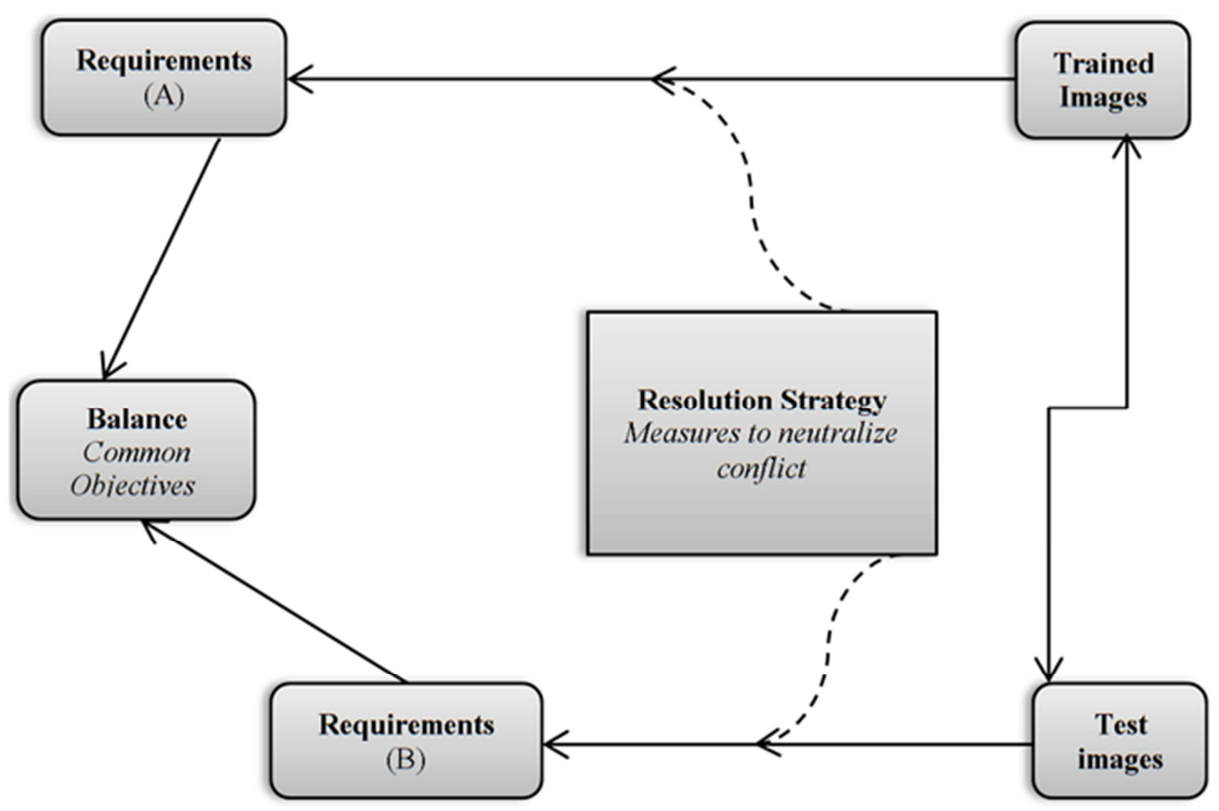

Figure 5. Facial Image Conflict Resolution Diagram.

\section{References}

[1] N. Eilidh and J. Rob, (2019). Deliberate Disguise In Face Identification. Journal of Experimental Psychology: Applied. ISSN 1076-898X. https://doi.org/10.1037/xap0000213

[2] N. Katz and K. McNulty, (1994). Conflict Resolution. http:// www.academia.edu.com. (Accessed: 15/01/2019)

[3] Z. Pawlak, and A. Skowron, (2006). Institute of Mathematics, Warsaw University, Banacha 2, 02-097, Warsaw, Poland.

[4] F. Al-Osaimi, M. Bennamoun and A. Mian, (2009). An Expression Deformation Approach To Non- Rigid 3d-Face Recognition. International Journal of Computer Vision. 81: 302-316. DOI 10.1007/s11263-008-0174-0.

[5] A. Jorstad, D. Jacobs and A. Trouve (2011). A Deformation and Lightening Insensitive Metric For Face Recognition
Based On Dense Correspondences. University of Maryland, College Park.

[6] N. Raajan, M. Ramkumar, B. Monisha, C. Jaiseeli and S. Prasanna (2012). Disparity Estimation From Stereo Images. International Conference on Modeling and Computing. (Elsevier) Procedia Engineering. 38 (2012) 462-472. www.sciencedirect.com.

[7] A. A. Alabi, (2013). A Modified Principal Component Analysis Technique For Recognizing African Bust. International Journal Of Engineering And Science (IJES); ||Volume||2 ||Issue|| 9||Pages|| 116-129||2013|| ISSN (e): 2319 1813 ISSN (p): 2319-1805.

[8] W. Kandlikar, T. Laxman and D. Jagannath (2014). Conversion of 2D image into 3D and Facial RecognitionBased Attendance System. International Journal of Advanced Research in Electrical, Electronics and Instrumentation Engineering (An ISO 3297: 2007 Certified Organization). Vol. 3 , Issue 6 . 
[9] A. Arpit, K. Abhishek and A. Leena (2014). Study on Advancement In Face Recognition Technology. Proceedings of National Conference on Recent Advances in Electronics and Communication Engineering (RACE-2014).

[10] A. A. Alabi, L. A. Akanbi and A. A. Ibrahim, (2015). Performance Evaluation of the Eigenface Algorithm on PlainFeature Images in Comparison with Those of Distinct Features. American Journal of Signal Processing, 5 (2): 32-39 DOI: $10.5923 / \mathrm{j}$.ajsp.20150502.02.

[11] F. Li., L. Xu, A. David and S. Clausi, (2015). Feature Extraction for Hyperspectral Imagery Via Ensemble Localized Manifold Learning. IEEE Geoscience And Remote Sensing Letters, Vol. 12, No. 12.

[12] J. Kumari, R. Rajesh. and K. Pooja, (2015). Facial Expression Recognition: A survey. (Elsevier) Procedia Computer Science, 58 (2015) 486 - 491. www.sciencedirect.com.

[13] K. Jain and H. Pann, (2017). Efficient Disparity Estimation From Stereo Images Using Hybrid- Guided Filter. The Imaging Science Journal, Volume 66, Issue 3. https://doi.org/10.1080/13682199.2017.1385893.

[14] A. TeixeiraLopes, E. Aguiar, A. De Souza and T. Santos (2017). Facial Expression Recognition with Convolutionary Neural Network Coping With Few Data and Training Sample Order. Pattern Recognition, Volume 61, Pages 610-628.
[15] S. Iloh and H. BongKang, (2018). Development And Utilization Of A Disgusting Image Dataset to Understand and Predict Visual Disgust. Image and Vision Computing, Volume 72, Pages 24-38.

[16] S. Yixin, J. Liu and Du L. (2018). Wrong Matching Point Elimination after Scale Invariant Transformation and Its Application in Image Machinery. Pattern Recognition and Image Analysis. Volume 28, Issue 1, pp. 87-96.

[17] C. Barker, L. Cox, J. Krinsky and G. Nilsen, (2013). Marxism and Social Movements. Konikelijke Brill NV. ISBN 978-9004-21175-9.

[18] Jameson (2014). Game Theory and Its Application. St. Catherine University, Sophia.

[19] J. Chappello (2019). Matching Pennies. https:// www.investopedia.com $/$ terms $/ \mathrm{m} /$ matching-pennies.asp

[20] M. Kress, J. Caulkins, G. Feichtinger, D. Grass and A. Seidl, (2017). Lanchester Model for Three-way Combat. Operations Research Department, Naval Postgraduate School, Monterey, California.

[21] N. Perry, (2009). Fractal Effects in Lanchester Models of Combat. Department of Defense, Australia. 